\title{
Colorless DQPSK Receiver for Wavelength Routed Packet-Switched Networks
}

Jensen, Jesper Bevensee; Osadchiy, Alexey Vladimirovich; Tafur Monroy, Idelfonso; Jeppesen, Palle

Published in:

I E E E Photonics Technology Letters

Link to article, DOI:

10.1109/LPT.2008.2004694

Publication date:

2008

Document Version

Publisher's PDF, also known as Version of record

Link back to DTU Orbit

Citation (APA):

Jensen, J. B., Osadchiy, A. V., Tafur Monroy, I., \& Jeppesen, P. (2008). Colorless DQPSK Receiver for Wavelength Routed Packet-Switched Networks. I E E E Photonics Technology Letters, 20(21-24), 1839 - 1841. https://doi.org/10.1109/LPT.2008.2004694

\section{General rights}

Copyright and moral rights for the publications made accessible in the public portal are retained by the authors and/or other copyright owners and it is a condition of accessing publications that users recognise and abide by the legal requirements associated with these rights.

- Users may download and print one copy of any publication from the public portal for the purpose of private study or research.

- You may not further distribute the material or use it for any profit-making activity or commercial gain

- You may freely distribute the URL identifying the publication in the public portal 


\title{
Colorless DQPSK Receiver for Wavelength Routed Packet-Switched Networks
}

\author{
Jesper Bevensee Jensen, Alexey V. Osadchiy, Idelfonso Tafur Monroy, and Palle Jeppesen
}

\begin{abstract}
We propose and demonstrate experimentally a scheme for the demodulation of 21.4-Gb/s return-to-zero differential quaternary phase-shift keying signals in packet-switched wavelength routed networks where packets at different wavelengths are arriving to the same demodulator. The idea is based on wavelength conversion, and in the demonstration, all channels were received error-free after wavelength conversion. In a packet arrival emulation, the ability of handling incoming packets at different wavelengths were successfully demonstrated.
\end{abstract}

Index Terms-Packet-switched, 060.2310 fiber optics, 060.4259 networks, 060.5060 phase modulation.

\section{INTRODUCTION}

I $\mathrm{N}$ packet-switched networks using wavelength routing, packets at different wavelengths will arrive to the same receiver at different times. For phase-modulated signals using delay demodulation, this is a problem due to the wavelength-dependent phase off-set in the demodulator. Using one demodulator for each incoming wavelength is a complex and costly, and therefore, undesirable solution. Tuning of the demodulator on a packet by packet rate requires fast control and feedback which would prove difficult to realize in practice. A more promising solution to the problem, would be to wavelength convert all incoming packets to one common wavelength.

In this letter, we propose and investigate experimentally, a method of converting a number of incoming phase-modulated signals to one common wavelength to which the receiver is optimized. The modulation format used in the experiments was 21.4-Gb/s return-to-zero (RZ) differential quaternary phase-shift keying (DQPSK), and the proposed scheme was demonstrated for three channels. The wavelength conversion was done through degenerate four-wave mixing (FWM) in nonlinear media. Experiments using highly nonlinear fiber (HNLF) as well as semiconductor optical amplifier (SOA) were performed. In both cases, error-free performance after wavelength conversion was achieved.

In order to add network functionality such as the routing information to an optical add-drop multiplexer to the scheme, a simple 1-bit optical label was added to the signal in the form of a weak sinusoidal amplitude modulation at a frequency equal to half the symbol rate. The use of the half-clock frequency for labelling ensures minimum label-induced signal degradation [1].

Manuscript received April 22, 2008; revised June 27, 2008. First published August 22, 2008; current version published October 31, 2008.

The authors are with the DTU Fotonik, Department of Photonics Engineering, Technical University of Denmark, Dk-2800 Kgs. Lyngby, Denmark (e-mail: jj@com.dtu.dk).

Color versions of one or more of the figures in this letter are available online at http://ieeexplore.ieee.org.

Digital Object Identifier 10.1109/LPT.2008.2004694
The proposed scheme is based on wavelength conversion by degenerate FWM with one pump laser for each signal laser. Appropriate allocation of pump and signal wavelengths ensures that the lasers which act as pumps for the wavelength conversion at the receiver side can be used as signal lasers for transmission to other network nodes. This can be seen by the following argument.

For degenerate FWM with $\omega_{s}>\omega_{p}$, where $\omega_{s}$ and $\omega_{p}$ are the angular frequencies of the pump and signal, the relations between the frequencies of the pump, signal, and FWM products are $\omega_{+}=2 \omega_{s}-\omega_{p}$ and $\omega_{-}=2 \omega_{p}-\omega_{s}$, where $\omega_{+}$and $\omega_{-}$are the angular frequencies of the up- and down-converted signals. If $\omega_{s}<\omega_{p}$, the relations become $\omega_{+}=2 \omega_{p}-\omega_{s}$ and $\omega_{-}=2 \omega_{s}-\omega_{p}$ [2]. For the phase, the corresponding relations become $\phi_{+}=2 \phi_{s}-\phi_{p}$ and $\phi_{-}=2 \phi_{p}-\phi_{s}$ for $\omega_{s}>\omega_{p}$, and $\phi_{-}=2 \phi_{s}-\phi_{p}$ and $\phi_{+}=2 \phi_{p}-\phi_{s}$ for $\omega_{s}<\omega_{p}$. Here, $\phi_{s}, \phi_{p}, \phi_{+}$, and $\phi_{-}$are the phases of the signal, pump up-converted and down-converted signals, respectively, and a common phase off-set has been omitted for clarity since only the differential phase between adjacent symbols is important due to the delay demodulation [2]. From the phase relations, it is seen that for $\omega_{s}>\omega_{p}$, apart from an inversion the data information contained in $\omega_{s}$ is preserved for the down-converted FWM product, whereas for $\omega_{s}<\omega_{p}$ the data information is preserved (apart from an inversion) for the up-converted FWM product. From the relation between the frequencies, it is seen that if the signal spacing is twice the pump spacing, the desired wavelength conversion of all signals to one common receiver wavelength can be achieved. If, on the other hand, two receiver wavelengths, and hence the requirement of two receivers are accepted, a double set can be constructed, where the pumps and signals have the same spacing. In this way, the possibility of re-using all lasers as both pumps and signals has been achieved, and no extra lasers have been added to the system due to the wavelength conversion. A double-set which would fit the $50-\mathrm{GHz}$ ITU grid is illustrated in Fig. 1.

\section{EXPERIMENTAL SETUP}

A simplified block diagram of the setup used in the experiment is shown in Fig. 2. Three distributed-feedback (DFB) lasers (tunable in 0.01-nm steps) were used for the signal and three external cavity lasers (ECLs) (tunable in 0.001-nm steps) were used as pumps for the wavelength conversion. As it is assumed that only packets at a single wavelength will be present at a given time, only one signal pump pair is active at the same time. Continuous-wave (CW) light from the three signal lasers were coupled into a Mach-Zehnder modulator (MZM) driven by an electrical clock signal at the pulse rate of $10.7 \mathrm{GHz}$ for pulse carving, and by a weak sinusoidal modulation at half the symbol rate $(5.35 \mathrm{GHz})$ for the label. After pulse-carving, 21.4-Gb/s DQPSK modulation was applied by 

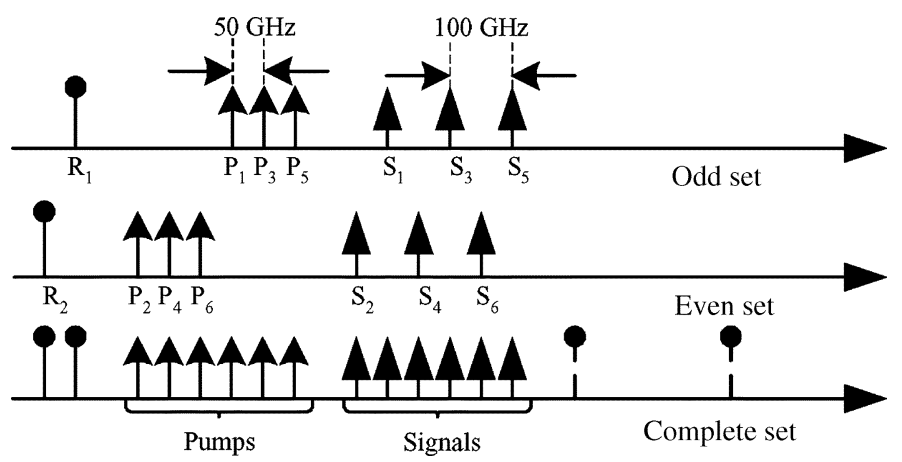

Fig. 1. Pump/signal allocation scheme in frequency domain. The dashed lines display the receiver frequencies in the case where pumps and signals are interchanged.
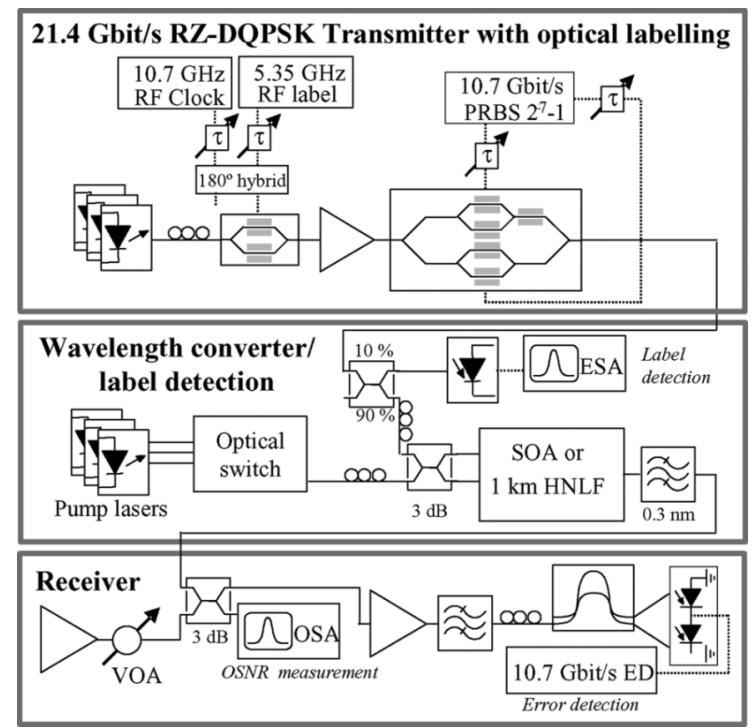

Fig. 2. Simplified block diagram of the setup used in the experiments.

a parallel MZM superstructure driven by two decorrelated 127-bit-long 10.7-Gb/s pseudorandom bit sequences (PRBS). Modulator loss was compensated by an erbium-doped fiber amplifier (EDFA). The 5.35-GHz labelling tone was detected by a photo diode and an electrical spectrum analyzer. This was done before the wavelength conversion, in order for the label to be used to control an optical add-drop multiplexer (OADM). In a real implementation, label processing could be performed by narrow electrical filtering around $5.35 \mathrm{GHz}$ followed by decision gating.

Before detection, conversion to the receiver wavelength was performed by FWM in either $1 \mathrm{~km}$ of HNLF or in an SOA. The pump laser corresponding to the wavelength of the incoming signal was selected by a fiber-optic switch (crosstalk $\simeq-80 \mathrm{~dB}$, switching-time $\simeq 300 \mathrm{~ms}$ ). Pump and signal powers at the input of the HNLF were 14.0 and $12.5 \mathrm{dBm}$, respectively. For the SOA, $12.0 \mathrm{dBm}$ was used for the pump and $9.0 \mathrm{dBm}$ for the signal. The signal was detected by a preamplified receiver setup, where the signal was loaded with noise from an open-ended EDFA before optical signal-to-noise ratio (OSNR) measurement by an optical spectrum analyzer, and data recovery by a one-symbol delay interferometer and a pair of balanced photodiodes. Bit-error ratio (BER) was
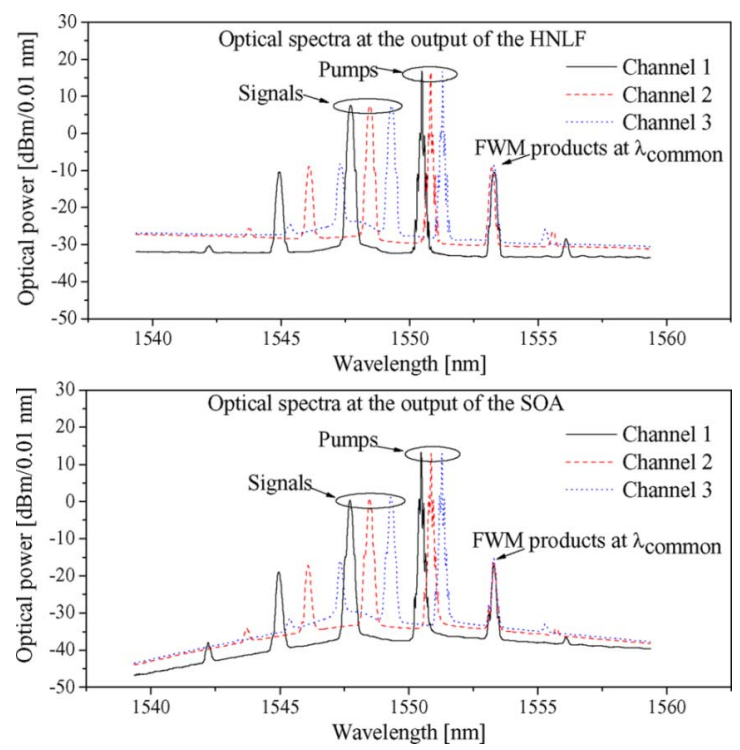

Fig. 3. Optical spectrum at the output of the HNLF and SOA showing pumps, signals, and FWM products for all three channels.

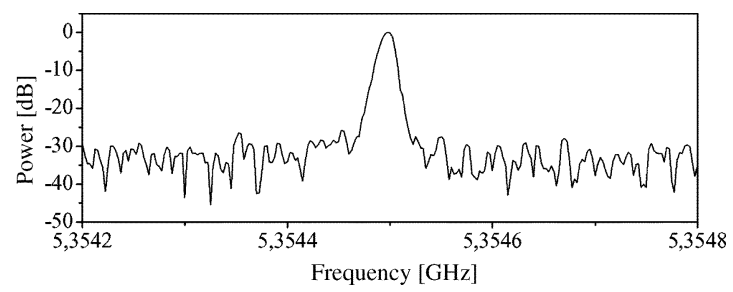

Fig. 4. Electrical spectrum (normalized to peak power) showing the label-tone at $5.35 \mathrm{GHz}$.

counted using an error detector programmed with the expected DQPSK tributary. In order to detect the two DQPSK tributaries simultaneously, two sets of delay interferometers and balanced photodiodes are required. In the laboratory implementation, the two tributaries were measured one at a time, and the total BER was calculated as the average of the two.

\section{RESULTS}

The optical power spectra at the output of the HNLF and SOA, respectively, are plotted in Fig. 3 for all three channels. As expected, the up-converted FWM product for all channels coincide on the same wavelength, thus verifying the wavelength conversion of three channels to one receiver wavelength. The nonflat noise floor in the SOA case is due to the nonflat gain spectrum of the SOA.

The electrical spectrum around $5.35 \mathrm{GHz}$ showing the optical label is plotted in Fig. 4, indicating a better than 20 -dB electrical signal-to-noise ratio for the label.

The measured BER of all channels with and without label is plotted in Fig. 5 before (a) and after wavelength conversion in the HNLF (b) and the SOA (c). In neither case was an indication of an error-floor observed, and the label induced no degradation of the BER. The required OSNR for a BER of $10^{-9}$ in the case without the label are given in Table I. After wavelength conversion in the HNLF, a small OSNR penalty of $0.7 \mathrm{~dB}$ for Channels 1 and 2, and 1.6 dB for Channel 3 is observed. For the SOA, the measured conversion penalties were 5.4, 5.6, and 

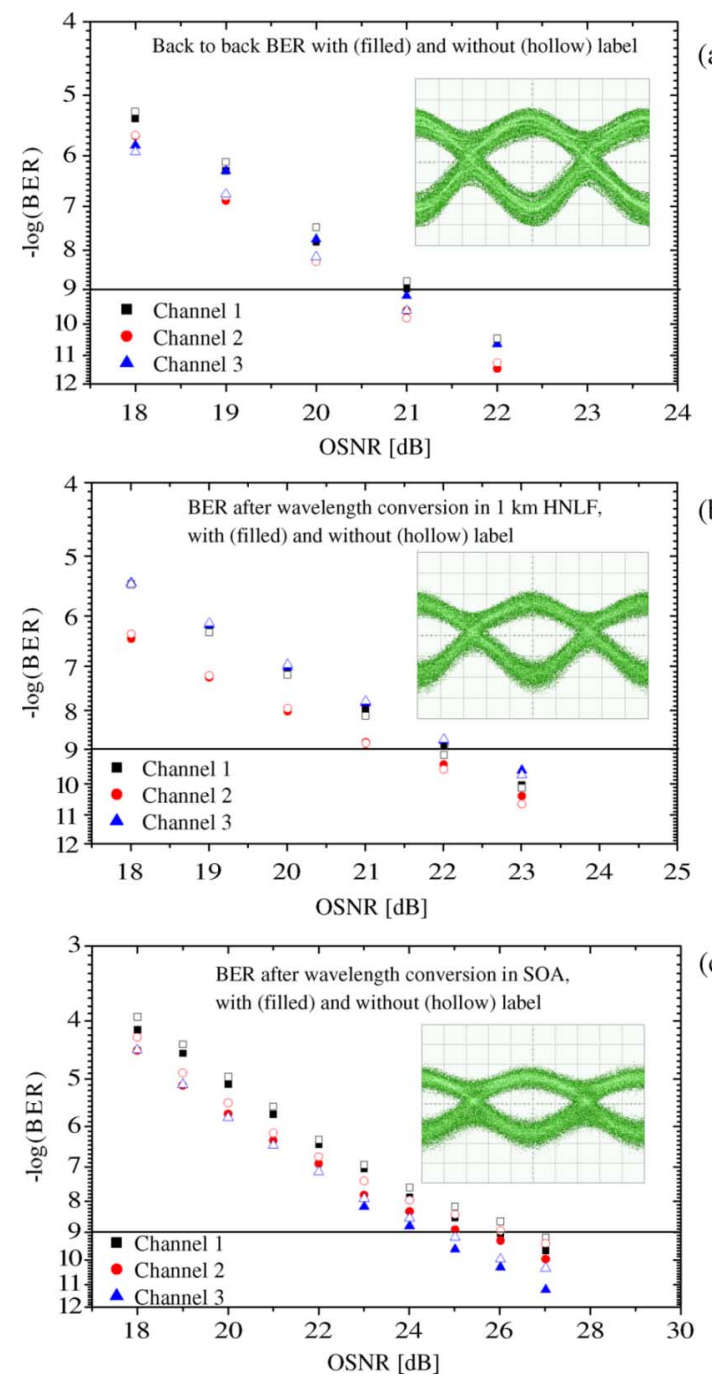

(c)

Fig. 5. BER versus OSNR (a) before, (b) after wavelength conversion in 1-km HNLF, and (c) after wavelength conversion in an SOA. Insets show the demodulated eye diagrams.

TABLE I

OSNR REQUIREMENTS FOR A BER OF $10^{-9}$ BACK-TO-BACK (B2B), AND AFTER WAVELENGTH CONVERSION IN HNLF AND SOA, RESPECTIVELY

\begin{tabular}{|c|c|c|c|}
\hline & Channel 1 & Channel 2 & Channel 3 \\
\hline B2B & $21.1 \mathrm{dBm}$ & $20.5 \mathrm{dBm}$ & $20.6 \mathrm{dBm}$ \\
\hline HNLF & $21.8 \mathrm{dBm}$ & $21.2 \mathrm{dBm}$ & $22.2 \mathrm{dBm}$ \\
\hline SOA & $26.5 \mathrm{dBm}$ & $26.1 \mathrm{dBm}$ & $24.7 \mathrm{dBm}$ \\
\hline
\end{tabular}

$4.1 \mathrm{~dB}$ for the three channels, respectively. The higher penalty for the SOA compared to the HNLF is confirmed by the eye diagram, which has a lower amplitude for the SOA. This is in good agreement with Fig. 3, which shows a lower OSNR for the signal after wavelength conversion in the SOA than in the HNLF. The SOA used in the experiment was a Mach-Zehnder structure chosen for availability rather than good FWM performance. Since only one arm of the device was used, but the noise generated in the other arm still degrades the signal, better performance would be expected from a device specifically designed for wavelength conversion. In order to emulate the scenario of
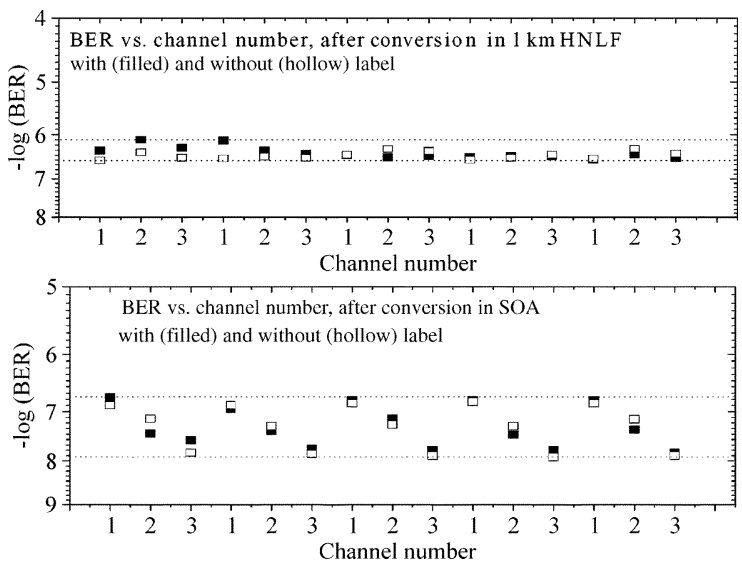

Fig. 6. Monitored BER in the packet arrival emulation experiment.

packets from different channels arriving to the same receiver at different times, an experiment was carried out where the active signal and pump pair was cyclically switched through the three channels, while monitoring the BER. The OSNR into the receiver was kept at $19 \mathrm{~dB}$ for the HNLF and at $23 \mathrm{~dB}$ for the SOA. The results are plotted in Fig. 6. For the SOA as well as for the HNLF, the variations in BER were very small. For the SOA, BER between $2 \times 10^{-7}$ and $3 \times 10^{-8}$ was measured, and for the HNLF between $9 \times 10^{-7}$ and $4 \times 10^{-7}$. Again, no degradation was observed from the label.

The method of wavelength conversion of packets with different wavelengths to one common wavelength has thus been demonstrated as a candidate for mitigation of the problem of wavelength-dependent receiver for phase-modulated signals. Fine-tuning of the wavelength of the pump lasers is required. It was found that $0.01-\mathrm{nm}$ steps for the signal lasers and $0.001-\mathrm{nm}$ steps for the pump lasers were sufficient to achieve error-free performance after wavelength conversion.

\section{CONCLUSION}

A receiver setup capable of coping with the problem of phase-modulated signals with different wavelengths arriving to the same receiver at different times in packet-switched networks using phase modulation has been investigated experimentally. Wavelength conversion of three incoming 21.4-Gb/s RZ-DQPSK signals at different wavelengths to one common wavelength was achieved with error-free reception of the wavelength converted signal. The wavelength conversion was performed by FWM in HNLF and in SOA. A small OSNR penalty of $0.7-1.6 \mathrm{~dB}$ was measured after conversion in the HNLF, 4.1- to 5.6-dB OSNR penalty was found after conversion in the SOA. A simple 1-bit optical label was added to the signal with no additional penalty.

\section{REFERENCES}

[1] I. T. Monroy, J. V. Olmos, M. G. Larrode, T. Koonen, and C. D. Jimnez, "In-band 16-QAM and multi-carrier SCM modulation to label DPSK payload signals for IP packet routing," Opt. Express, vol. 14 pp. 1000-1005, 2006.

[2] G. T. Zhou, K. Xu, C. Yan, Y. Su, and J. T. Lin, "Self-pumping wavelength conversion for DPSK signals and DQPSK generation through four-wave mixing in highly nonlinear optical fiber," IEEE Photon. Technol. Lett., vol. 18, no. 22, pp. 2389-2391, Nov. 15, 2006. 